\title{
DESAMBIGUAÇÃO TAXONÔMICO-NOMENCLATURAL E TIPIFICAÇÃO DAS ESPÉCIES DO GRUPO SEALYANA (AMARYLLIDACEAE, AMARYLLIDOIDEAE), E SEUS SINÔNIMOS ${ }^{1}$
}

\author{
HENRIQUE MALLMANN BÜNEKER ${ }^{2}$ REGIS EDUARDO BASTIAN ${ }^{3}$
}

\begin{abstract}
RESUMO
É fornecida uma revisão nomenclatural dos táxons do grupo Sealyana e propostas tipificações. Este grupo é constituído por dois gêneros monoespecíficos, Eithea (E. blumenavia) e Eusarcops (E. reticulata), sendo que neste artigo é proposto o restabelecimento do último. São discutidas as relações morfológicas e filogenéticas dos táxons deste grupo dentre as Hippeastrinae, apresentando-se uma chave para identificação dos gêneros desta subtribo que ocorrem no Brasil.
\end{abstract}

Palavras-chave: Hippeastreae, Hippeastrinae, Eithea, Eitea blumenavia, Eusarcops, Eusarcops reticulata.

\section{ABSTRACT}

[Taxonomic-nomenclatural disambiguation and typification of species of the Sealyana group (Amaryllidaceae, Amaryllidoideae), and their synonyms].

A nomenclatural revision of the taxa belonging to the Sealyana group is provided and typifications proposed. This group consists of two monospecific genera, Eithea (E. blumenavia) and Eusarcops (E. reticulata), and in this article the reestablishment of the latter is proposed. Morphological and Phylogenetic relationships of the taxa of this group among the Hippeastrinae are discussed, presenting a key for identification of the genera of this subtribe that occur in Brazil.

Key words: Hippeastreae, Hippeastrinae, Eithea, Eitea blumenavia, Eusarcops, Eusarcops reticulata.

\section{INTRODUÇÃO}

Dentre os tratamentos taxonômicos clássicos para a atual família Amaryllidaceae J. SaintHilaire (1805: 134), subfamília Amaryllidoideae Burnett (1835: 446), do clado hippeastróide (sensu Meerow et al., 2000), uma grande diversidade de posicionamentos diferentes já foram propostos em hierarquias variadas (e.g. Baker, 1878; 1888; Bentham, 1883; Pax \& Hoffman, 1930; Traub \& Uphof, 1938; Traub \& Uphof, 1940; Traub \& Moldenke, 1949; Traub, 1958; Traub, 1980; Traub, 1983). Com o advento das ferramentas de biotecnologia relacionadas essencialmente a genética, filogenias baseadas em

1 Recebido em 13-V-2016 e aceito para publicação em 30-V-2016.

2 Técnico em Paisagismo e acadêmico do curso de Engenharia Florestal, Universidade Federal de Santa Maria. henriquebuneker@mail.ufsm.br

3 Biólogo do Centro de Reprodução de Espécies Raras do Brasil (CRER Brasil). crerbrasil@hotmail.com caracteres moleculares vieram a lume e, novas propostas para circunscrições taxonômicas, sobretudo genéricas, de alguns táxons de posicionamentos duvidosos, têm sido amplamente discutidas (e.g. Flagg et al., 2010; García et al., 2014; Meerow, 2010; Meerow et al., 2000; Oliveira, 2012; Ravenna, 2003; Traub, 1963).

Apesar da atenção sistemática que a tribo Hippeastreae Herbert ex Sweet (1829: t. 14) recebeu, e ainda tem recebido, pela sua importância horticultural, principalmente através de análises filogenéticas recentes baseadas em caracteres moleculares (Oliveira, 2012; García et al., 2014), uma estabilidade taxonômico-genérica ainda não foi estabelecida principalmente devido à falta de caracteres morfológicos diagnósticos e a significante homoplasia. Esta última uma característica comum de Amaryllidoideae (Meerow et al., 1999), o que provavelmente, na subtribo Hippeastrinae Walpers (1852: 616), seja reflexo de sua história 
evolutiva reticulada, tema que tem sido amplamente abordado visto que marcadores moleculares nucleares e plastidiais apresentam histórias evolutivas diferentes, gerando incongruências consensuais que limitam suas aplicações taxonômico-nomenclaturais (Oliveira, 2012; García et al., 2014). Além disto a grande homoplasia, em geral, leva a busca por caracteres autopomórficos ao insucesso e, torna difícil, as próprias delimitações morfológicas intergenéricas. É o caso observado em Hippeastrinae onde as circunscrições genéricas precisam ser definidas por um conjunto mútuo de caracteres morfológicos, pois estes isoladamente analisados não passam de sinapomorfias.

Ao decorrer de várias décadas têm-se evidenciado a incerteza do posicionamento taxonômico das espécies, de Hippeastrinae (sensu García et al., 2014) que apresentam sementes (não discoides) e folhas atípicas (oblanceolado-decorrentes, pseudopecioladas). As espécies que compartilham estas excepcionalidades morfológicas possuem uma longa história de recombinações nomenclaturais pelos seus difíceis posicionamentos dentro das circunscrições convencionais já propostas para a maioria dos gêneros de Hippeastrinae. Apesar das afinidades morfológicas entre os dois táxons em questão, cujos basiônimos são Amaryllis reticulata L'Héritier (1788: 8) e Griffinia blumenavia Koch \& Bouche ex Carrière (1867: 32), os estudos filogenéticos recentes (Oliveira, 2012; García et al., 2014) revelaram que se tratam de duas linhagens distintas, sendo as relações morfológicas existentes resultado de convergência, ou mais provavelmente resultado de introgressão genética pretérita de linhagens distintas com consequências morfológicas dentro de uma complexa história evolutiva.

Traub (1938) concebeu uma circunscrição ampla para o gênero Amaryllis Linnaeus (1753: 292) e foi o primeiro autor a considerar a grande afinidade morfológica entre os dois táxons em questão, propondo um subgênero para abrigar estas espécies, Amaryllis subgen. Sealyana Traub (1938: 131), constituído por duas espéci- es A. blumenavia (Koch \& Bouche ex Carrière) Traub (1938: 131), definida com espécie tipo, e que foi na ocasião transferida para o gênero Amaryllis e A. reticulata. Décadas mais tarde Meerow et al. (2000) apresentou uma filogenia para as Amaryllidaceae, Amaryllidoideae americanas baseada em sequências de nrDNA ITS, revelando que o então Hippeastrum blumenavium (Koch \& Bouche ex Carrière) Sealy (1937: t. 9504) constituía uma linhagem muito distante das demais espécies de Hippeastrum amostradas, sugerindo que se considerasse $H$. blumenavium como gênero distinto de Hippeastrum, isso dando suporte a posterior proposição do gênero Eithea Ravenna (2002: 2). O posicionamento taxonômico do basiônimo Amaryllis reticulata não foi um caso diferente, visto que por mais de uma vezes foi proposta como distinta dos demais gêneros por ocasião aceitos, sendo considerado espécie tipo dos gêneros Leopoldia Herb. e Eusarcops Raf., passando por uma diversidade de recombinações para ouros gêneros, como também seus sinônimos.

Assim, como estas duas entidades taxonômicas não se enquadram dentre as circunscrições morfológico-filogenéticas atuais dos convencionais gêneros de Hippeastrinae (sensu García et al., 2014), aqui são tratadas ambas como espécies que constituem gêneros monoespecíficos distintos, compostos por Eusarcops reticulata (L'Héritier) Rafinesque (1838: 11) e Eithea blumenavia (Koch \& Bouche ex Carrière) Ravenna (2002: 4). Englobando ambos os gêneros em um grupo morfológico, podemos informalmente chamar este de grupo Sealyana. O objetivo deste artigo, por fim, é a desambiguação taxonômiconomenclatural destes táxons incluindo o reestabelecimento de Eusarcops como gênero, vista a necessidade de uma sinopse nomenclatural para compilação dos sinônimos e combinações destes, e também suas tipificações que permanecem indefinidas até esta data. Para subsidiar a identificação morfológica destes táxons, neste artigo também é apresentada uma chave 
para identificação dos gêneros que constituem a subtribo Hippeastrinae no Brasil.

\section{NOMENCLATURA E TAXONOMIA}

1. Eusarcops Raf., Flora Telluriana, v. 4, p. 11, 1838.

Tipo: Eusarcops reticulata

$=$ Leopoldia Herb., Transactions of the Horticultural Society of London, v. 4, p. 181, 1822. nom. rejic. para legitimar Leopoldia Parl., Flora Palermitana, v. 1, p. 435, 1845. (Asparagaceae Juss.), nom. cons.

Observações nomenclaturais: A identidade dos gêneros Amaryllis e Hippeastrum foram tema de profundas discussões e, apenas a poucas décadas atingiram uma relativa estabilidade taxonômico-nomenclatural (Traub, 1983; Goldblatt, 1984), endossada pela aceitação da tipificação de uma planta sul africana como Amaryllis belladonna L. no XIV Congresso Internacional de Botânica de 1987 transcorrido em Berlin, Alemanha (Meerow et al., 1997). Como consequência desta tipificação, o gênero Hippeastrum passou a abrigar todas as dezenas de espécies americanas que eram tratadas como Amaryllis e, o último passou a ser constituído exclusivamente de espécies sul africanas, sendo representado por duas espécies, $A$. belladonna L. e A. paradisicola Snijman \& Williamson. Herbert (1822) prioritariamente propôs a criação do gênero Leopoldia Herb., sendo este constituído por duas espécies, $L$. reticulata e $L$. striatifolia, sendo este gênero, para o autor, próximo de Coburgia Herb. pela morfologia das sementes e próximo de Amaryllis pelas flores, entretanto este nome foi rejeitado para legitimar Leopoldia Parl., nom. cons. (Asparagaceae Juss.). Posteriormente Rafinesque (1838) propôs Eusarcops como novo gênero, o diferenciando de Amaryllis principalmente por apresentar estigma clavado, cápsulas carnosas e sementes globosas de coloração negra e envolvidas por arilo avermelhado, sendo a espécie tipo E. reticulata. Com a rejeição de Leopoldia Herb. o princípio da prioridade indica que Eusarcops seja o nome para o gênero em questão vista sua validade e legitimidade.

Observações taxonômicas: As espécies que constituem os gêneros do grupo Sealyana podem ser facilmente reconhecidas dentre as demais Hippeastrinae pelas folhas largamente oblanceolado-decorrentes, pseudopecioladas, ou quase, e sementes globosas, elipsoidicas ou ovoides. Eusarcops tem como principais características diagnósticas quando comparado a Eithea, gênero ao qual é afim, o estigma clavadocapitado ou brevemente lobado e hipanto alongado com mais de $15 \mathrm{~mm}$ de comprimento. Durante as últimas décadas Eusarcops tem sido considerado sinônimo de Hippeastrum, que é um dos gêneros de maior diversidade específica em Hippeastreae. Todavia das mais de 60 espécies que compõem Hippeastrum, Eusarcops pode ser facilmente diferenciado morfologicamente pelas folhas e sementes (folhas largamente oblanceolado-decorrentes, pseudopecioladas ou quase e sementes globosas, elipsoidicas ou ovoides $v s$. folhas estreitamente oblanceoladas a oblongo-lineares, sésseis e sementes discoides). Cabe salientar que algumas espécies de Hippeastrum como por exemplo: $H$. vittatum (L'Hér.) Herb., H. elegans (Spreng.) H.E. Moore, H. brasilianum (Traub \& L.J. Doran) Dutilh, H. goiamum (Ravenna) Meerow e H. canastrense J. Dutilh \& R.S. Oliveira, possuem assim como Eusarcops reticulata hipanto longo, o que pode gerar algumas confusões na delimitação intergenérica de Eusarcops e, identificações errôneas principalmente em espécimes de herbários quando este caractere é observado isoladamente. Não sendo esta uma característica exclusiva de Eusarcops, mas sim, provavelmente, resultado de convergência, ou resultado do processo de hibridações pretéritas na sua história evolutiva.

Em termos filogenéticos, baseando-se em caracteres moleculares, uma ampla discussão tem sido levantada, visto que evidentemente a evolução de Hippeastreae é reticulada (Oliveira, 2012; García et al., 2014). Comparando-se 
as árvores filogenéticas baseadas em marcadores plastidiais e nucleares apresentadas por García et al., (2014), observam-se incongruências relacionadas ao posicionamento de Eusarcops. Nestas árvores filogenéticas, derivadas da análise de sequências ITS nrDNA e cpDNA, podemos observar que o clado em questão, composto pelo terminal "Hippeastrum reticulatum", ora constitui uma linhagem irmã do clado formado pelas demais espécies amostradas de Hippeastrum típicos (marcador ITS nrDNA) e, ora forma um clado juntamente com Eithea blumenavia (marcador cpDNA), este numa linhagem irmã do grupo de algumas espécies de Sprekelia e Zephyranthes. Isso leva a hipótese de que, concebendo apenas um evento de hibridação, no passado, a linhagem que deu origem a Eusarcops reticulata seja próxima da linhagem que deu origem a Eithea, tendo ocorrido nesta, introgressão genética da linhagem que deu origem a Hippeastrum, sendo a última afirmação evidenciada pela análise dos dados de marcadores plastidiais e do cariótipo destes táxons. Por fim, vistas as evidências morfológicas e filogenético-moleculares apresentadas e discutidas neste tópico, acredita-se que a melhor alternativa consensual para o táxon em questão seja o reestabelecimento do gênero Eusarcops, como distinto e monotípico, constituído por Eusarcops reticulata.

1.1 Eusarcops reticulata (L'Hér.) Raf., Flora Telluriana, v. 4, p. 11, 1838.

= Amaryllis agatha Steud., Nomenclator Botanicus, ed. 2, v. 1, p. 72, 1840. nem. nud.

= Amaryllis praeclara Sweet, Loudon's Hortus Britannicus, p. 506, 1830. nem. nud.

$=$ Amaryllis princeps Vell., Florae Fluminensis, v. 3, p. 130, 1825. Tipo: Lectótipo (aqui designado): Ilustração original de Amaryllis princeps Vell., Florae Fluminensis, v. 3, t. 115, 1827. (Fig. 3).

= Leopoldia princeps (Vell.) M.Roem., Familiarum Naturalium Regni Vegetabilis Synopses Monographicae, v. 4, p. 129, 1847. comb. illeg.
$=$ Amaryllis principis Salm-Dyck, Nova Acta Physico-medica Academiae Caesareae Leopoldino-Carolinae Naturae Curiosorum Exhibentia Ephemerides sive Observationes Historias et Experimenta, v. 10, p. 154, 1821. Tipo: Lectótipo (aqui designado): Ilustração original de Amaryllis principis Salm-Dyck, Nova Acta Physico-medica Academiae Caesareae Leopoldino-Carolinae Naturae Curiosorum Exhibentia Ephemerides sive Observationes Historias et Experimenta, v. 10, t. 9, 1821. (Fig. 4).

$$
\text { = Leopoldia principis (Salm-Dyck) }
$$

M.Roem., Familiarum Naturalium Regni Vegetabilis Synopses Monographicae, v. 4, p. 128, 1847. comb. illeg.

= Amaryllis caroliniae Sterler, Hortus Nymphenburgensis, seu, Enumeratio plantarum in Horto Regio Nymphenburgensi cultarum, p. 44, 1826.

= Amaryllis reticulata L'Hér., Sertum Anglicum, p. 8, 1788. Tipo: Lectótipo (aqui designado): Planta cultivada no Reino Unido: J. Lee, s.n., s.d. (G!) (Fig. 1).

= Callicore reticulata (L'Hér.) Link, Handbuch zur Erkennung der nutzbarsten und am häufigsten vorkommenden Gewächse, v. 1, p. $193,1829$.

= Coburgia reticulata (L'Hér.) Herb., Curtis's Botanical Magazine, t. 2113 (5), 1819. = Coburgia reticulata (L'Hér.) Herb., An Appendix, p. 34, 1821.

= Hippeastrum reticulatum (L'Hér.) Herb., Curtis's Botanical Magazine, t. 2475 (2), 1824.

= Leopoldia reticulata (L'Hér.) Herb., Transactions of the Horticultural Society of London, v. 4, p. 181, 1822. nom. rejic.

$=$ Amaryllis reticulata var. striatifolia Herb., Curtis's Botanical Magazine, t. 2113, 1820. Tipo: Lectótipo (aqui designado): Ilustração original de Amaryllis reticulata var. striatifolia Herb., Curtis's Botanical Magazine, t. 2113, 1820. (Fig. 5).

= Amaryllis striatifolia (Herb.) Sweet, Loudon's Hortus Britannicus, p. 506, 1830. 


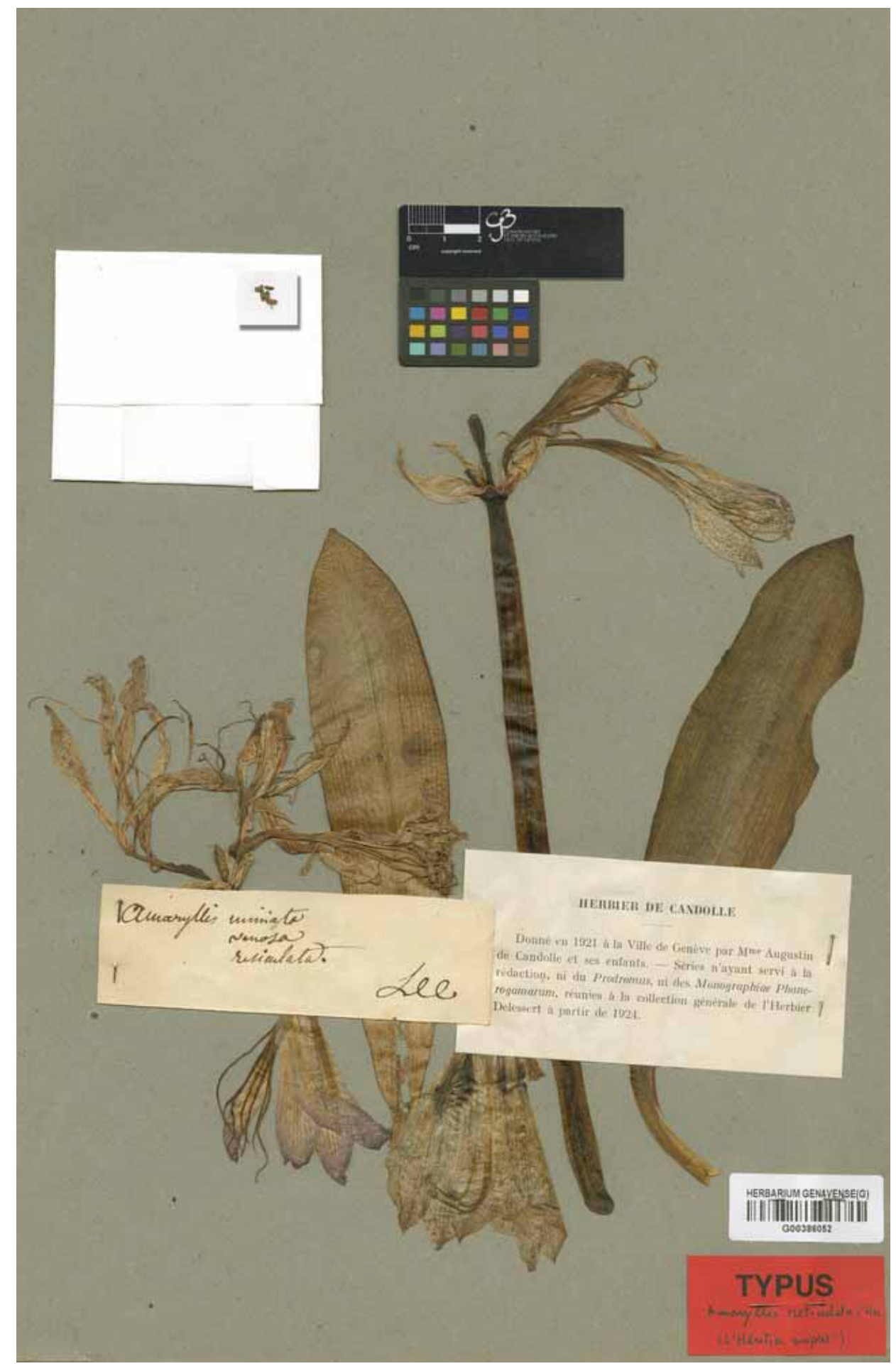

FIGURA 1 - Exsicata coletada por J. Lee s.n., s.d. de Amaryllis reticulata L'Hér. (aqui designado como lectótipo). (créditos da digitalização: herbário G, Bercode G00386052). 


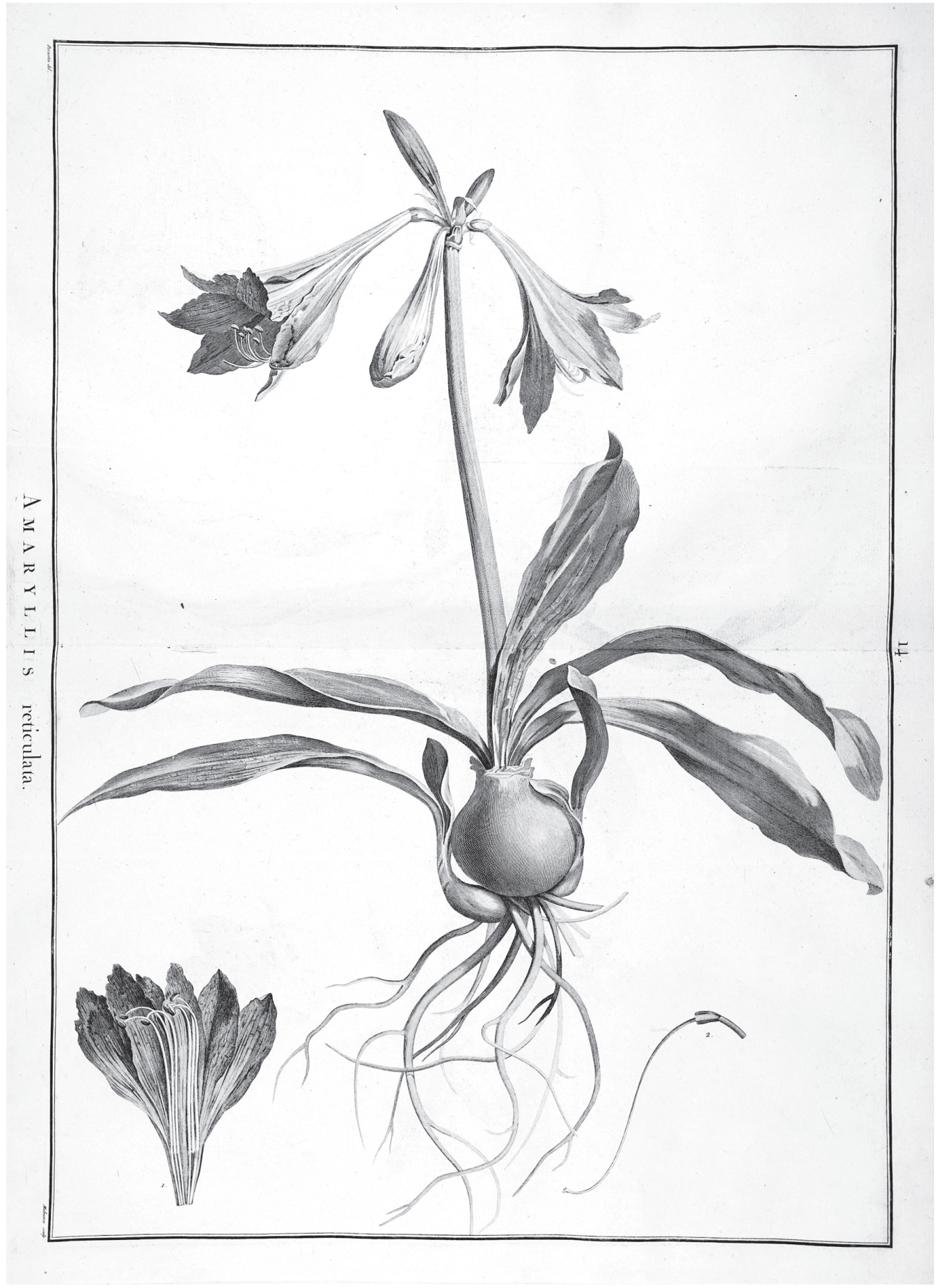

FIGURA 2 - Ilustração original de Amaryllis reticulata L'Hér. da obra Sertum Anglicum, t. 14, 1788 (créditos da digitalização: Missouri Botanical Garden, Peter H. Raven Library). 


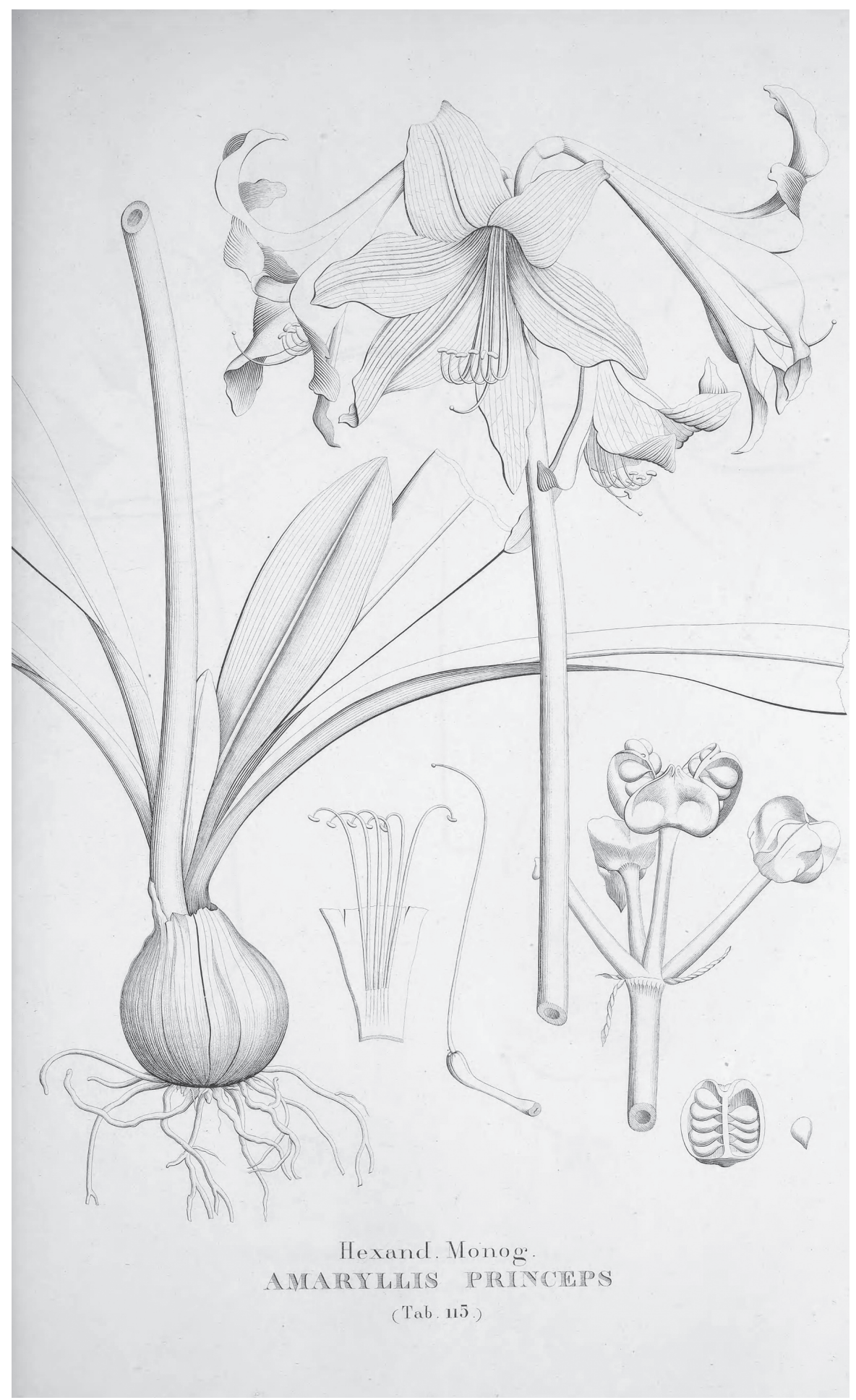

FIGURA 3 - Ilustração original de Amaryllis princeps Vell. (aqui designado como lectótipo) da obra Florae Fluminensis, v. 3, t. 115, 1827 (créditos da digitalização: Smithsonian Libraries). 


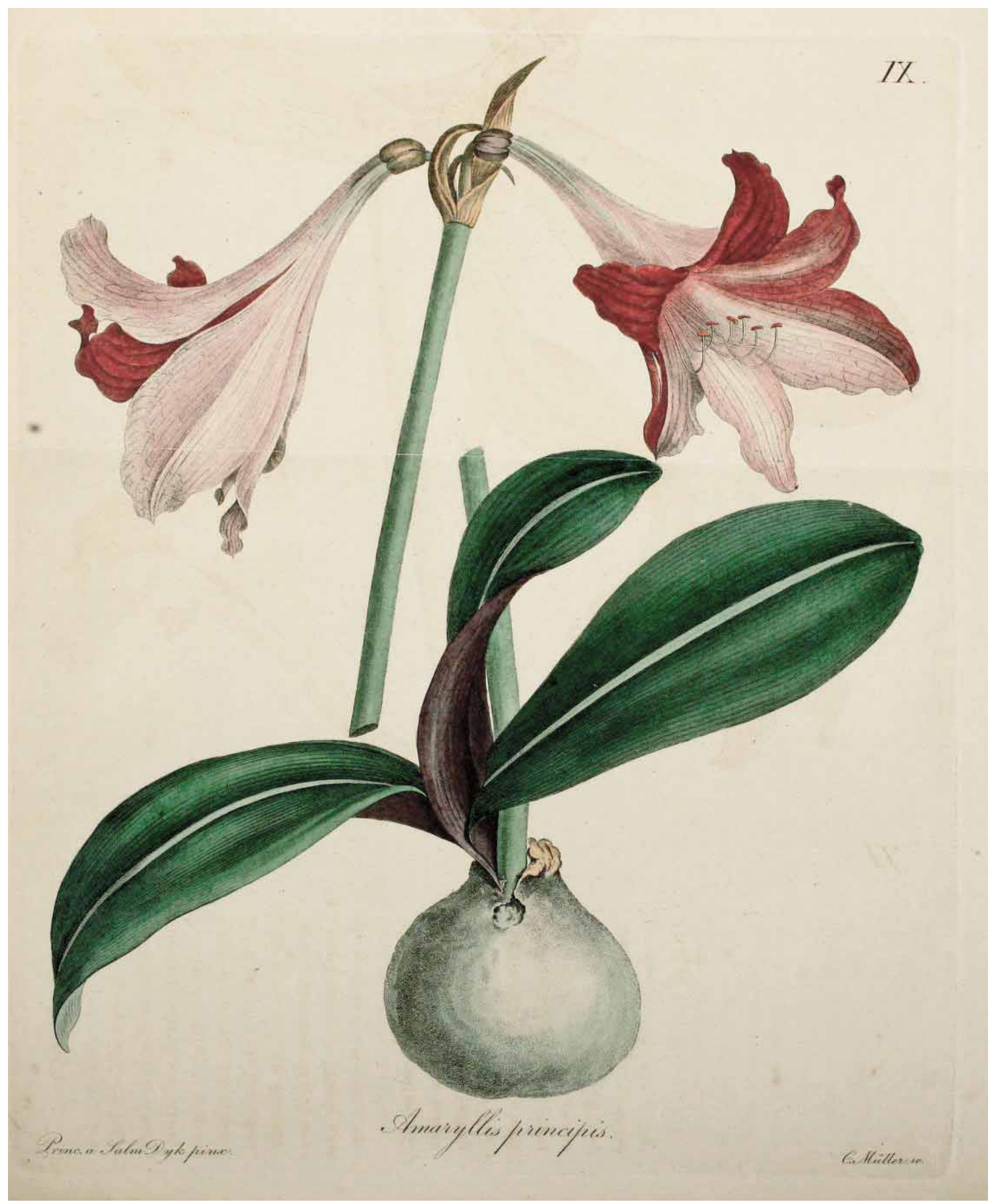

FIGURA 4 - Ilustração original de Amaryllis principis Salm-Dyck (aqui designado como lectótipo) da obra Nova Acta Physico-medica Academiae Caesareae Leopoldino-Carolinae Naturae Curiosorum Exhibentia Ephemerides sive Observationes Historias et Experimenta, v. 10, t. 9, 1821, 1827 (créditos da digitalização: Natural History Museum Library, London). 


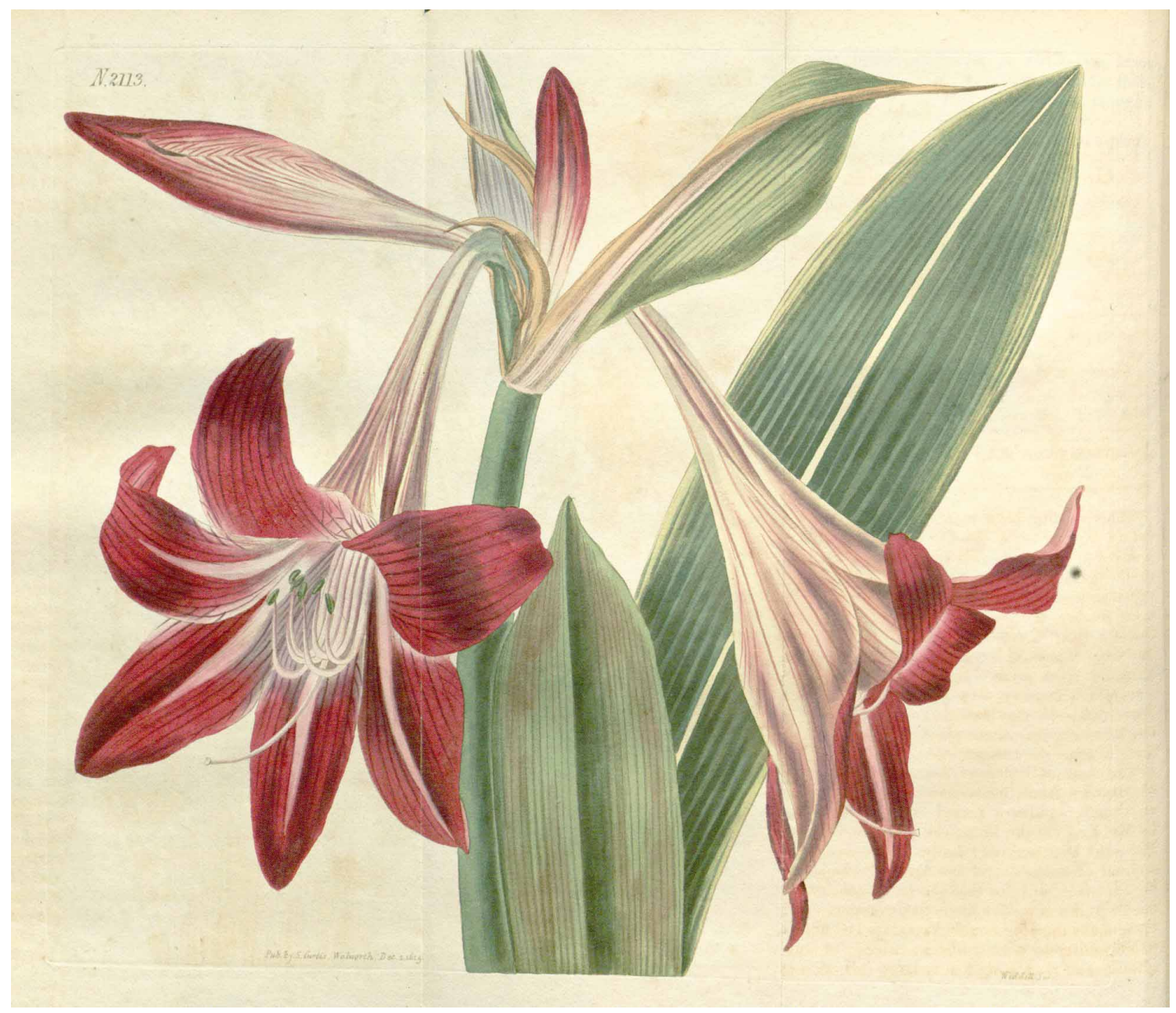

FIGURA 5 - Ilustração original de Amaryllis reticulata var. striatifolia Herb. (aqui designado como lectótipo) da obra Curtis's Botanical Magazine, t. 2113, 1820 (créditos da digitalização: Missouri Botanical Garden, Peter H. Raven Library). 
= Coburgia reticulata var. striatifolia (Herb.) Herb., Curtis's Botanical Magazine, t. 2113 (5), 1820.

= Hippeastrum striatifolium (Herb.) Herb., Curtis's Botanical Magazine, t. 2475 (2), 1824.

= Hippeastrum reticulatum var. striatifolium (Herb.) Herb., Amaryllidaceae, p. 137, 1837.

= Leopoldia striatifolia (Herb.) Herb., Transactions of the Horticultural Society of London, v. 4, p. 181, 1822. nom. rejic.

= Amaryllis sweetii Herb. ex Sweet, Loudon's Hortus Britannicus, p. 506, 1830. nom. nud.

= Hippeastrum latifolium M.Roem., Familiarum Naturalium Regni Vegetabilis Synopses Monographicae, v. 4, p. 140, 1847. Tipo: desconhecido

= Hippeastrum wolteri Wittm., Gartenflora, v. 44, p. 201, 1895. Tipo: Lectótipo (aqui designado): Ilustração original de Hippeastrum wolteri Wittm., Gartenflora, v. 44, t. 49, 1895.

Observações nomenclaturais: Originalmente o binômio Amaryllis reticulata foi descrito por L'Héritier ${ }^{4}$ em 1788 e posteriormente uma grande diversidade de combinações e sinônimos foram propostos revelando o difícil posicionamento taxonômico da espécie. Vários destes sinônimos foram aqui lectotipificados pelas suas ilustrações originas, o que expõem a referência necessária para as suas manutenções como sinonímia. O tipo de Amaryllis reticulata, basiônimo de Eusarcops reticulata, permaneceu por muitas décadas desconhecido, sendo que Oliveira (2012) relata que Herbert (1821) indicou como lectótipo de Coburgia reticulata (sinônimo de Eusarcops reticulata) a ilustração apresentada em Curtis's botanical magazine, v.

${ }^{4}$ Charles Louis L'Héritier de Brutelle (1746-1800) foi um magistrado e botânico francês. Morreu assassinado, deixando um herbário com cerca de 8.000 espécimes e uma das mais importantes bibliotecas de botânica (Stafleu \& Cowan, 1986).

5 Augustin Pyrame de Candolle (1778-1841) foi um ilustre botânico suíço.
47, t. 2113 (1819). Esta tipificação, entretanto, é um neótipo, visto que o material indicado como tipo não tem relação direta com o material analisado e descrito pelo autor de Amaryllis reticulata. Segundo Stafleu \& Cowan (1986) parte das cerca de 8000 exsicatas da coleção de L'Héritier foram vendidas para A.P. de Candolle $^{5}$ que tornou estas a base do seu herbário privado que, atualmente, constitui o acervo histórico do Conservatoire et Jardin botaniques de la Ville de Genève (herbário G). Neste acervo que encontra-se o espécime que certamente foi utilizado por L'Héritier para descrição de Amaryllis reticulata (Fig. 1), isto evidenciado pela grande similaridade deste espécime com o ilustrado no seu protótipo (Fig. 2). Este espécime, proveniente da coleção de J. Lee $^{6}$, é aqui indicado como lectótipo do basiônimo Amaryllis reticulata.

Cabe salientar que um dos sinônimos da espécie em questão, H. latifolium, apesar de apresentar uma descrição idêntica a $E$. reticulata que o sustenta como sinônimo, permanece com seu tipo desconhecido. Ainda sobre este binômio observam-se algumas inconsistências como um dos seus sinônimos, Amaryllis rutila var. latifolia Mart. nom. nud., nome que efetivamente nunca foi publicado entretanto consta em Martius (1837) como sinônimo de Amaryllis fulgida Ker., sendo assim um nomen dubium.

Observações taxonômicas: A pesar da afinidade floral de Eusarcops reticulata com espécies de Hippeastrum que apresentam hipanto longo (e.g. $H$. vittatum, $H$. elegans, $H$. brasilianum, $H$. goiamum e $H$. canastrense J.Dutilh \& R.S.Oliveira), este pode ser facilmente distinguido pelas folhas largamente oblanceolado-decorrentes, pseudopecioladas, ou quase, e sementes globosas, elipsoidicas ou ovoides. Nas últimas décadas tem sido amplamente aceito pela comunidade científica o seu

\footnotetext{
6 James Lee (1715-1795) foi um horticultor e viveirista britânico que introduziu ao cultivo na Europa plantas americanas.
} 
posicionamento, apesar de frágil, no gênero Hippeastrum, entretanto este é amplamente questionável visto o que é aqui discutido, não existindo uma base nem morfológica e nem filogenética explicita para a manutenção desta posição. A partir disto aqui é considerado como a única espécie representante do gênero Eusarcops.

2. Eithea Ravenna, Botanica Australis, v. 1, p. 2, 2002.

Tipo: Eithea blumenavia (K. Koch \& C.D. Bouché ex Carrière) Ravenna.

= Amaryllis subgen. Sealyana Traub, Herbertia, v. 5, p. 131, 1938. Tipo: Amaryllis blumenavia (K. Koch \& C.D. Bouché ex Carrière) Traub.

$=$ Griffiniopsis J.H. Dutilh \& A.W. Meerow, TEKTRAN, United States Department of Agriculture, Agricultural Research Service, 2002. Disponível em: < http:// www.nal.usda.gov/ttic/tektran/data/000012/82/ 0000128200.html>. nom. nud.

Observações nomenclaturais: Originalmente Sealyana foi descrita por Traub (1938) como subgênero de Amaryllis para abrigar as espécies que segundo o autor apresentam sementes pouco numerosas e carnosas ( $v s$. sementes numerosas e compressas nos subgêneros Phycella, Clinanthe, Rhodophiala, Macropodastrum, Lais, Aschamia e Omphalissa sensu Traub 1938). A. blumenavia foi definida claramente como espécie tipo de Amaryllis subgen. Sealyana, também sendo citada $A$. reticulata como pertencente a este subgênero. Em obra posterior Traub \& Moldenke (1949) erroneamente citaram A. reticulata como espécie tipo de Amarylis subgênero Sealyana. Em 2002 Dutilh \& Meerow divulgaram que iriam descrever Griffiniopsis, um novo gênero para abrigar a espécie que na ocasião era aceita sob combinação de Hippeastum blumenavium, entretanto, no mesmo ano, Ravenna descreveu o gênero Eithea para abrigar este táxon, sendo Griffiniopsis não descrito oficialmente e atual- mente considerado nomem nudum. Vê-se que tais proposições genéricas condizem com as normas definidas pelo vigente Código Internacional de Nomenclatura para Algas, Fungos e Plantas, mesmo que Amaryllis subgen. Sealyana poderia ter sido elevada a gênero, pois leva em conta o princípio de que um nome não tem prioridade fora do ranque em que foi publicado (capítulo II, seção 3, artigo 11.2).

Observações taxonômicas: $\mathrm{O}$ gênero Eithea possui, assim como o gênero Eusarcops, características morfológicas em comum que as reúnem, mesmo que artificialmente, em grupo, Sealyana. Estas características podem ser facilmente reconhecidas dentre as demais Hippeastrinae, sendo as folhas largamente oblanceolado-decorrentes, pseudopecioladas ou quase e sementes globosas, elipsoidicas ou ovoides. Eithea pode ser diferencia de Eusarcops por apresentar estigma trífido e hipanto curto com até $7 \mathrm{~mm}$ de comprimento.

Apesar das dúvidas que no passado existiam com relação ao posicionamento taxonômico de Eithea, pela sua afinidade morfológica com espécies do gênero Griffinia, atualmente sua posição na tribo Hippesatreae já está consolidada visto que o gênero Griffinia pertence a tribo Griffineae juntamente com o gênero Worsleya (W. Watson ex Traub) Traub, posição que tem sido amplamente aceita pela comunidade científica mundial e que tem sido fortemente suportada por estudos filogenéticos baseados em caracteres moleculares (Meerow et al., 2000; Oliveira, 2012; García et al., 2014).

2.1 Eithea blumenavia (Koch \& Bouche ex Carrière) Ravenna, Botanica Australis, v. 1, p. 4, 2002.

$$
\text { = Griffinia blumenavia Koch \& Bouche }
$$
ex Carrière, Revue Horticole, v. 39, p. 32, 1867. Tipo: Lectótipo (aqui designado): Ilustração original de Griffinia blumenavia Koch \& Bouche ex Carrière, Revue Horticole, v. 39, p. (32-)tabula(-33), 1867. (Fig. 6). 


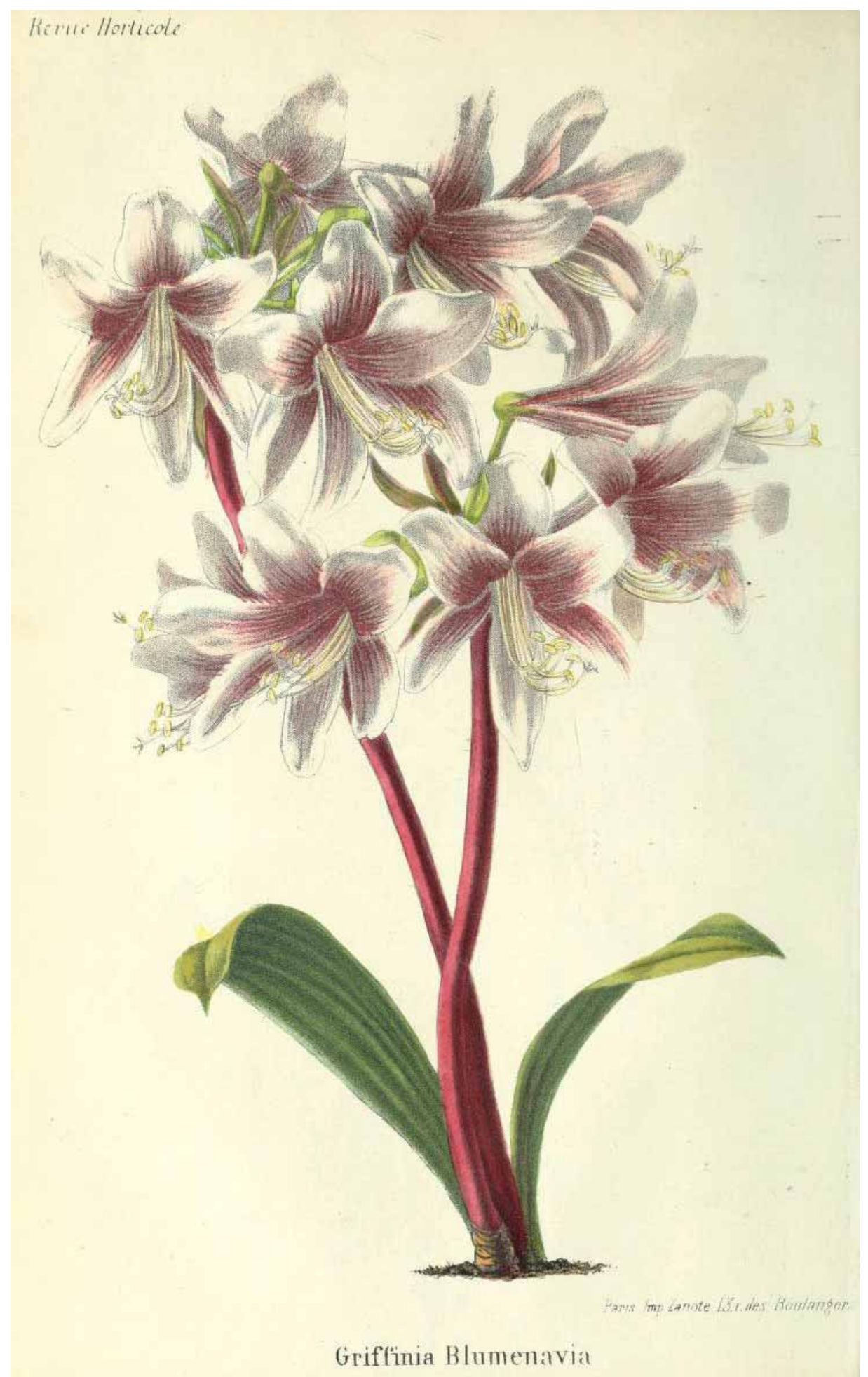

FIGURA 6 - Ilustração original de Griffinia blumenavia Koch \& Bouche ex Carrière (aqui designado como lectótipo) da obra Revue Horticole, v. 39, p. (32-)tabula(-33), 1867 (créditos da digitalização: Harvard University Botany Libraries). 
$=$ Amaryllis blumenavia (Koch \& Bouche ex Carrière) Traub, Herbertia, v. 5, p. 131, 1938.

$=$ Griffiniopsis blumenavia (Koch \& Bouche ex Carrière) J.H. Dutilh \& A.W. Meerow, TEKTRAN, United States Department of Agriculture, Agricultural Research Service, 2002. Disponível em: < http:// www.nal.usda.gov/ttic/tektran/data/000012/82/ 0000128200.html>. comb. illeg.

= Hippeastrum blumenavium (Koch \& Bouche ex Carrière) Sealy, Curtis's Botanical Magazine, t. 9504. 1937.

= Hippeastrum iguapense $\mathrm{R}$. Wagner, Wiener Illustrirte Garten-Zeitung, v. 28, p. 281, 1903. Tipo: desconhecido.

= Amaryllis iguapensis (R. Wagner) Traub \& Uphof, Herbertia, v. 5, p. 125, 1938.

Observações nomenclaturais: Carrière (1867) originalmente descreveu e ilustrou Griffinia blumenavia atribuindo a autoria do binômio a C. Koch e Bouché. O primeiro baseando-se em um espécime cultivado no Jardim Botânico de Berlim, proveniente da "ilha de Santa Catarina"7 (Brasil) e enviado pelo Dr. H. Blumenau $^{8}$, que relatou que se tratava de uma planta comumente encontrada na localidade. Posteriormente recombinado para Amaryllis blumenavia e Hippeastrum blumenavium, este táxon originalmente definido como espécie tipo de Amaryllis subgen. Sealyana, entretanto
Eithea prevalece sobre este conforme já comentado neste artigo. Apesar dos diversos tratamentos taxonômicos e combinações nomenclaturais pelas quais esta espécie já passou, a tipificação desta nunca foi abordada, vista a falta de espécimes depositados em herbário analisados pelos seus autores, que é o caso geral de Amaryllidaceae descritas nos séculos passados, é aqui definido como lectótipo a ilustração da espécie apresentada na sua obra original (Fig. 6).

Eithea blumenavia possui apenas um sinônimo (cuja obra original rara e tipo, não foram analisados pelos autores de artigo), Hippeastrum iguapense R. Wagner, sendo esta sinonimização, proposta por Traub (1938), amplamente aceita.

Observações taxonômicas: Eithea blumenavia é a única espécie que, oficialmente, compõem o gênero, Eithea. Seu hábito geral, mas principalmente o vegetativo (folhas pseudopecioladas e oblanceolado-decorrentes), explica a sua descrição originalmente no gênero Griffinia, visto que são características comuns a estes gêneros. Estas características morfológicas a tornam facilmente reconhecível em comparação as demais espécies contidas nos gêneros de Hippeastrinae, mesmo quando comparada a Eusarcops reticulata, sua mais próxima afinidade morfológica. Uma descrição muito rica de sua morfologia e alguns dados anatômicos podem ser encontrados em Ravenna (2002).

Chave para identificação de gêneros da subtribo Hippeastrinae (Amaryllidaceae, Amaryllidoideae, Hippeastreae) que ocorrem no Brasil ${ }^{9}$

1a. Folhas largamente oblanceolado-decorrentes, pseudopecioladas ou quase.

Sementes globosas, elipsoidicas ou ovoides (grupo Sealyana) 2

1b. Folhas estreitamente oblanceoladas a oblongo-lineares, sésseis. Sementes discoides ..... 3

2a. Estigma clavado-capitado ou brevemente lobado. Hipanto com mais de $15 \mathrm{~mm}$ de comprimento (Mata Atlântica; SC, PR, SP, MG, RJ, ES e BA) ${ }^{10}$ Eusarcops Raf.

7 A ilha de Santa Catarina é parte do município de Florianópolis, no estado de Santa Catarina, Brasil.

8 Hermann Bruno Otto Blumenau (1819-1899) foi um filósofo, administrador e químico farmacêutico alemão e o fundador do município de Blumenau, no estado de Santa Catarina (Brasil).
9 Chave baseada na observações de espécimes in habitat, em cultivo e herborizados e, nas descrições apresentadas na literatura especializada.

${ }^{10}$ (Bioma no qual a espécie ocorre; sigla dos estados em que ocorrem espécies do gênero em questão do sul ao norte respectivamente). 
2b. Estigma trífido. Hipanto com até $7 \mathrm{~mm}$ de comprimento (Mata Atlântica; SC, PR e SP)

3a. Inflorescência reduzida a uma única flor. Flor na antese ereta e com hipanto menor que $2 \mathrm{~cm}$ de comprimento

3b. Inflorescência com 1-14 flor(es). Flor(es) na antese reflexa(s), patente(s), subereta(s) ou se ereta obrigatoriamente solitária e com hipanto maior que $7 \mathrm{~cm}$ de comprimento

4a. Escapo inconspícuo de tamanho muito reduzido, subterrâneo (Pampa; RS)

Haylockia Herb. ${ }^{11}$

4b. Escapo conspícuo, desenvolvido, alongado, aéreo (Pampa, Mata Atlântica,

Cerrado, Pantanal, Caatinga e Floresta Amazônica; RS, SC, PR,

SP, RJ, MS, MG, GO, DF, MT, BA, TO, SE, AL,

PE, PI, PB, RN, CE e MA)

Zephyranthes Herb.

5a. Flor(es) na antese ereta(s), subereta(s) ou subpatente-ereta(s).

Inflorescência reduzida a uma única flor ou raramente com até 4 flores

5b. Flor(es) na antese patente(s) ou reflexa(s). Inflorescência geralmente

com 2 ou mais flores ou raramente reduzida a uma única flor

6a. Hipanto maior que $7 \mathrm{~cm}$ de comprimento. Inflorescência com 2-3

brácteas com ápice simples e estigma trífido ou se com uma bráctea

com ápice bífido obrigatoriamente apresentando estigma capitado

(Cerrado e Caatinga; MG, TO e BA)

Tocantinia Ravenna

6b. Hipanto menor que $4 \mathrm{~cm}$ de comprimento. Inflorescência

com uma bráctea com ápice bífido e estigma trífido

(Pampa, Mata Atlântica, Cerrado, Pantanal, Caatinga e Floresta

Amazônica; RS, SC, PR, SP, RJ, MS, MG, GO, DF, MT, BA,

TO, SE, AL, PE, PI, PB, RN, CE e MA)

Habranthus Herb.

7a. Folhas menos largas que $1 \mathrm{~cm}$, planas; Inflorescência

com 3-8(-14) flores (Pampa; RS) Rhodophiala C. Pres $1^{12}$

7b. Folhas mais largas que $1 \mathrm{~cm}$, acanaladas ou se menos largas que $1 \mathrm{~cm}$ e planas apresentando obrigatoriamente inflorescência com 1-2(-3) flores (Pampa, Mata Atlântica, Cerrado, Pantanal, Caatinga e Floresta Amazônica; todos os estados brasileiros).

Hippeastrum Herb.

${ }^{11}$ Haylockia Herb. é um gênero que carece de uma revisão toxonômico-nomanclatural pois durante várias décadas este ficou obscuro vista a falta de elucidação relacionada aos táxons que foram transferidos para este. Neste artigo ele é considerado distinto do gênero Zephyranthes, ao qual tem permanecido sinonimizado nas últimas décadas, pois apesar das muitas similaridades morfológicas, diversos estudos filogenéticos baseados em caracteres moleculares têm o evidenciado como distinto (e.g. García et al., 2014) e, morfologicamente, existe sustentação para este ser diferenciado. No Brasil é representado por uma espécie, Haylockia americana (Hoffmanns.) Herter, que tem sido na atualidade amplamente conhecida como Zephyranthes americana Ravenna.

${ }^{12}$ Rhodophiala é um gênero essencialmente andino que, até a poucos anos atrás, possuía, citadas para o Brasil, ocorrência de duas espécies, $R$. bifida (Herb.) Traub e $R$. cipoana Ravenna. Entretanto a última foi transferida para o gênero Hippeastrum, agora sendo $H$. cipoanum (Ravenna) Meerow. 


\section{AGRADECIMENTOS}

Ao pessoal da Library of Congress (National Library of the United States), pela digitalização de algumas obras vitais à confecção deste artigo.

\section{REFERÊNCIAS BIBLIOGRÁFICAS}

SAINT-HILAIRE, J.H.J. Exposition des families naturelles, v. 1. Treuttel \& Würtz, Paris, 1805. $512 \mathrm{p}$.

BURNETT, G.T. Outlines of Botany. John Churchill, Princes-street, Soho, London, 1835. 1190 p.

MEEROW, A.W.; GUY, C.L.; LI, Q.-B.; YANG, S.L. Phylogeny of the American Amaryllidaceae based on nrDNA ITS sequences. Systematic Botany, v. 25, p. 708-726, 2000.

BAKER, J.G. An enumeration and classification of the species of Hippeastrum. Journal of Botany, v. 16, p. $79-85,1878$.

BAKER, J. G. Handbook of Amaryllideae. George Bell \& Sons. London, 1888. 216 p.

BENTHAM, G. Amaryllidaceae. In: BENTHAM, G.; HOOKER, J.D. (eds.). Genera Plantarum, v. 3 , n. 2, p. 711-740. London. 1883.

PAX, F.; HOFFMAN, K. Amaryllidaceae. In: ENGLER, A.; PRANTL, K. Die Natürlichen Pflanzenfamilien. Leipzig: Wilhelm Engelmann, 1930. p. 391-430.

TRAUB, H.P.; UPHOF, J.C. Tentative Revision of the genus Amaryllis (Linn. ex parte) (syn. Hippeastrum Herb.). Herbertia, v. 5, p. 114-131, 1938.

TRAUB, H.P.; UPHOF, J.C. Critical Review of Sealy's "Amaryllis and Hippeastrum". Herbertia, v. 6, p. 163-166, 1940.

TRAUB, H.P.; MOLDENKE, H.N. Amaryllidaceae: Tribe Amarylleae. The American Plant Life Society, La Jolla, 1949.

TRAUB, H.P. The Amaryllis Manual. New York: The Macmillan Company, 1958.

TRAUB, H.P. The Subgenera of the Genus Amaryllis. Plant Life, v. 36, p. 43-45, 1980.

TRAUB, H.P. The lectotypification of Amaryllis belladonna L. (1753). Taxon, v. 32, p. 253-267, 1983.

FLAGG, R.O.; SMITH, G.L.; MEEROW, A.W. New combinations in Habranthus (Amaryllidaceae) in Mexico and Southwestern U.S.A. Novon, v. 20, p. 33-34, 2010.
GARCÍA, N.; MEEROW, A.W.; SOLTIS, D.E.; SOLTIS, P.S. Testing deep reticulate evolution in Amaryllidaceae tribe Hippeastreae (Asparagales) with ITS and chloroplast sequence data. Systematic Botany, v. 39, p. 75-89, 2014.

MARTIUS C.F.P. VON. Hypoxideae, Burmanniaceae, Haemodoraceae, Vellosiaceae, Pontederiaceae, Hydrocharideae, Alismaceae, Butomaceae, Juncaceae, Rapateceae, Liliaceae, Amaryllideae In: Flora Brasiliensis (C.F.P. Martius; A.G. Eichler, eds.). Leipzig, v.3, part. 2, p. 49-164, 1837.

MEEROW, A.W. Convergence or reticulation? Mosaic evolution in the canalized American Amaryllidaceae. In: Seberg, O.; Petersen, G.; Barfod, A.S.; Davis J.I. (Eds.) Diversity, phylogeny, and evolution in the monocotyledons. Aarhus University Press, Aarhus, 2010. p. 145168.

OLIVEIRA, R.S. DE O gênero Hippeastrum Herb. (Amaryllidaceae) no Brasil: evidência de evolução reticulada e análise de caracteres florais. Thesis for $\mathrm{PhD}$, Universidade Estadual de Campinas, Campinas, 2012. 240 p.

RAVENNA, P.F. Elucidation and systematics of the Chilean genera of Amaryllidaceae. Botanica Australis, v. 2, p. 1-20, 2003.

STAFLEU, F.A.; COWAN, R.S. Taxonomic literature. A selective guide to botanical publications and collections with dates, commentaries and types. Regnum Vegetabile, v. 105, p. 1-980, 1986.

TRAUB, H.P. The Genera of Amaryllidaceae. American Plant Life Society, La Jolla, California, 1963. 35p.

MEEROW, A.W.; FAY, M.F.; CHARLES, L.G.; LI, Q.-B.; ZAMAN, F.Q.; CHASE, M.W. Systematics of Amaryllidaceae based on cladistic analysis of plastid sequence data. American Journal of Botany, v. 86, p. 1325-1345, 1999.

WALPERS, G.G. Annales Botanices Systematicae, vol. 3. Sumtibus F. Hofmeister, Lipsiae, 1852. $1168 \mathrm{p}$.

RAVENNA, P.F. Eithea, a new genus of Brazilian Amaryllidaceae. Botanica Australis, v. 1, p. 1$8,2002$.

SWEET, R. Habranthus robustus. The British Flower Garden, containing coloured figures \& descriptions of the most ornamental and curious hardy flowering plants, series 2, t. 14, 1829. 
L'HÉRITIER, C.L. Sinopsis operis, Sertum Anglicum, p. 1-20, 1788.

CARRIÈRE, E.A. Griffinia blumenavia. Revue Horticole, v. 39, p. 32-33, 1867.

TRAUB, H.P. Subgenus Sealyana, genus Amaryllis (Linn. ex Parte) Uphof, Amaryllidaceae. Herbertia, v. 5, p. 131, 1938.

LINNAEUS, C. Species Plantarum v. 1, 1753. 560 p.

SEALY, J.R. Hippeastrum blumenavia, Curtis's Botanical Magazine, t. 9504. 1937.

RAFINESQUE, C.S. Flora Telluriana, v. 4, 1838. 135 p.
TRAUB, H.P. The lectotypification of Amaryllis belladonna L. (1753). Taxon, v. 32, n. 2, p. $253-$ 267, 1983.

GOLDBLATT, P. Proposal to conserve 1176 Amaryllis and typification of A. belladonna (Amaryllidaceae). Taxon, v. 33, n. 3, p. 511-516, 1984.

MEEROW, A.W.; VAN SCHEEPEN, J.; DUTILH, J.H.A. Transfers from Amaryllis to Hippeastrum (Amaryllidaceae). Taxon, v. 46, n. 1, p. 15-19, 1997. 